\title{
Peer Feedback Processes in the Game Industry
}

\author{
Joseph Seering* \\ Carnegie Mellon University \\ Pittsburgh, USA \\ jseering@cs.cmu.edu
}

\author{
Tianying Chen \\ Carnegie Mellon University \\ Pittsburgh, USA \\ tianyinc@andrew.cmu.edu
}

\author{
Ray Mayol* \\ Carnegie Mellon University \\ Pittsburgh, USA \\ rmayol@andrew.cmu.edu
}

\author{
Amy Cook \\ Carnegie Mellon University \\ Pittsburgh, USA \\ amyshann@andrew.cmu.edu
}

\author{
Erik Harpstead \\ Carnegie Mellon University \\ Pittsburgh, USA \\ eharpste@cs.cmu.edu
}

\author{
Jessica Hammer \\ Carnegie Mellon University \\ Pittsburgh, USA \\ hammerj@cs.cmu.edu
}

\begin{abstract}
A wide variety of design strategies, tools, and processes are used across the game industry. Prior work has shown that these processes are often collaborative, with experts in different domains contributing to different parts of the whole. However, the ways in which these professionals give and receive peer feedback have not yet been studied in depth. In this paper we present results from interviews with industry professionals at two game studios, describing the ways they give feedback. We propose a new, six step process that describes the full feedback cycle from making plans to receive feedback to reflecting and acting upon that feedback. This process serves as a starting point for researchers studying peer feedback in games, and allows for comparison of processes across different types of studios. It will also help studios formalize their understanding of their own processes and consider alternative processes that might better fit their needs.
\end{abstract}

\section{Author Keywords}

Game design; peer feedback; design processes; game studios

\section{CCS Concepts}

-Human-centered computing $\rightarrow \mathrm{HCI}$ theory, concepts and models; Empirical studies in $\mathrm{HCI}$; $\bullet$ Software and its engineering $\rightarrow$ Software design engineering;

\section{INTRODUCTION}

The HCI community has engaged at length with processes for design, working with industry practitioners and studying outcomes of different approaches [14]. These processes are core to the development of new ideas, concepts, and products $[6,17]$. While less work in the HCI community has focused

\footnotetext{
* Equal contribution

Permission to make digital or hard copies of all or part of this work for personal or classroom use is granted without fee provided that copies are not made or distributed for profit or commercial advantage and that copies bear this notice and the full citation on the first page. Copyrights for components of this work owned by others than the author(s) must be honored. Abstracting with credit is permitted. To copy otherwise, or republish, to post on servers or to redistribute to lists, requires prior specific permission and/or a fee. Request permissions from permissions@ acm.org.

CHI PLAY'19, October 22-25, 2019, Barcelona, Spain

(C) 2019 Copyright held by the owner/author(s). Publication rights licensed to ACM. ISBN 978-1-4503-6688-5/19/10 . \$15.00

DOI: https : //doi .org/10.1145/3311350. 3347176
}

specifically on game design processes, the field uses a range of approaches [7, 9, 24]. What these approaches have in common is that they are iterative, experiential, and interdisciplinary. Receiving feedback on prototypes is key to all three elements. Game professionals use feedback to iterate their designs, to discover the nature of the experience they have created, and to align a range of stakeholders within interdisciplinary teams.

Game design and software engineering processes, while related, face significantly different challenges. Per Murphy-Hill, Zimmerman, and Nagappan, "games generally have one and only one requirement - that they are 'fun.' " [18, p. 3]. This fun must emerge from a creative intermixing of art styles, user interfaces, level designs, sounds, and many more details.

In this work we explore processes that game industry professionals use for exchanging feedback with peers on their work. Within the context of our study, "work" might mean early concepts, specs, scripts, art, music, sound, storyboards, interactive prototypes, or any of the other core elements of game design and development. By "peers", we mean other industry professionals, which may mean co-workers at the same company or other professional contacts. Peer feedback is central to processes for learning, design, and development. Because game development is iterative, experiential, and interdisciplinary, understanding peer feedback is key to improving the game creation process as a whole.

Compared to playtesting [5], peer feedback has received relatively little attention within the games literature. In this work, we therefore draw on literature from engineering design, psychology, and learning science. This prior work has identified aspects of feedback processes that make them most effective, from characteristics of the person giving feedback [20] to characteristics of the feedback itself $[4,26]$. It has also identified challenges of peer feedback, such as making sense of a large body of feedback from peers $[26,28]$. We aim to build on this prior work to enhance our understanding of the role of peer feedback in existing industry game design processes, and to identify how the challenges of peer feedback play out in the game development context.

In this paper we present three core contributions. First, we present findings related to peer feedback from interviews with 
professionals at two game design studios. While these studios do engage in giving peer feedback, they had not formally established a model for these processes and how they fit into the development cycle. Per this point, we next present a formal model for understanding peer feedback processes in the game industry, which emerged from interview data. Finally, we discuss directions for future research.

The framework we present has implications for industry professionals, for game researchers, and for scholars of peer feedback. Our framework can help industry professionals become more aware of the strengths and weaknesses of their design choices around feedback, and help them consider alternative practices. Game researchers can use our framework to design interventions to study and improve the game design process; additionally, game researchers who also create games can apply this work to their own design processes. Finally, researchers who study peer feedback in other contexts can use our work to explore the impact of situational and structural differences between contexts. Across all three groups, we believe this work provides a foundation for exploring feedback and collaboration more broadly in game design processes.

\section{LITERATURE REVIEW}

In this section we review the relevant literature on the game design process and in feedback processes more generally.

\section{Feedback}

Feedback processes between peers and with advisors have been studied from a number of perspectives. In this work, we define peer feedback as when a person receives comments, thoughts, ideas, reactions, or other forms of feedback from collaborators and colleagues or gives feedback to them, specifically including both reciprocal and non-reciprocal interactions. There are several types of feedback defined in the literature, divided between the (non-exclusive) categories of feedback intended to change a person and feedback intended to help improve an artifact or design. Nelson and Schunn describe three types of the former: motivational feedback, which is aimed to influence beliefs and impact willingness to participate; reinforcement feedback, which serves to reward or to punish displayed behaviors; and informational feedback, which aims to change performance in a particular direction [19]. In this work, we focus instances where professionals receive feedback on what they have created or plan to create in order to facilitate its development and improvement, which is to some extent aimed to help the professional develop their skills but primarily focused on improving the created artifact.

Researchers, primarily working from within education and learning science, have identified best practices of feedback in general contexts. While some people tend to respond better to feedback from people in authority [20], qualities of good feedback exist, regardless of source. Successful feedback is relevant, copious, timely, and diverse [26]. It is also specific [8] and actionable [33]. Using positive-valenced language [20], and qualitative rather than quantitative language [4] makes feedback better received.

While anonymized feedback has some benefits, namely allowing a feedback provider to be sufficiently critical without fear of upsetting social dynamics [3], this anonymity requires careful management lest it lead to unproductive behaviors [31]. Feedback received is useful only when understood and reflected on. Students who receive helpful feedback can learn to improve their work [21], and designers can learn to make improvements on their project and process.

Feedback in engineering design literature may be included as one piece within the larger participatory design frameworks of collaborative [32, 35] and co-creative [2, 29] design, both of which connect consumers with producers during the design process. Though co-design extends beyond feedback between peers, its structure and challenges are similar to those we find in the process we elaborate for peer feedback here. These include gathering participants with sufficiently diverse sets of competencies to contribute and inform design and iterating through multiple cycles and "learning loops" [29]. In our work here we take a middle approach between focusing on specific details of individual pieces of feedback and outlining the design process as a whole, focusing on the roles and characteristics of peer feedback in the greater design process.

\section{Game Design Processes}

Game design is not a simple or straightforward activity; designers do not take an idea and work it until completion. Instead, game design is iterative, experiential, and interdisciplinary.

Iterative. Modern game design work is broken into parts [23]. Designers start with a working concept and build upon it through iterative cycles [9, 22]. Design thinking promotes failing fast and failing early. Knowing what works and what doesn't is crucial in order for designers to decide when they should push on and when they are wasting their time. Getting feedback rapidly makes for more iterative cycles, which often makes the game better and the designers better at what they do [36]. Designers typically have easy access to peers for feedback, which makes peer feedback an easy solution for rapid and iterative prototyping.

Experiential. Fun, a very important factor for games, is sometimes fleeting and fickle. Finding the fun in an experience is not a linear process, both because players are unpredictable and because game systems can have emergent properties that are difficult to predict $[9,24]$. Prior work interviewing game designers has shown that companies employ a variety of strategies in guiding development of the player's experience [11, 12]. Feedback about the game experience helps to confirm that fun is kept and the overall quality of the experience is maintained [10].

One of the ways game designers frequently seek user feedback is through playtesting, and though there are many methods for playtesting, Fullerton, Swain, and Hoffman note that they all have the common element of "gain[ing] useful feedback from players in order to improve your game" [9, pp. 196-197]. Even when a game works, designers still benefit from having new perspectives on their work. What may be accessible and familiar to them may not be the same for their consumers. Since consumers have levels of novelty, accessibility, and familiarity that they can tolerate, miscalculations can be costly [34]. Though playtesting is often seen as a way of getting 
feedback from potential users or customers, it can also be an internal process for peer feedback where members within and across teams can help provide feedback by playtesting works-in-progress. We detail our findings in a later section.

Interdisciplinary. Game development does not occur in a vacuum. Designers often work in teams, rather than individually, and teams may be connected to a community, a company, and other stakeholders. Also, a game development team might include designers, artists, programmers, writers, and/or people in other roles. The team as a whole might be producing one piece of a larger game, which makes them interdependent with other teams. Additionally, players, modders, and other community groups have a stake in the games the team produces. Each of these groups may wish to offer feedback throughout the development process to guide it according to their interests. However, experts in a given area frame problems differently from novices (e.g. [16]), use different language (e.g. [30]), and have different priorities. Game design places a higher value on creativity than other software disciplines, "requirements tend to be more subjective", and there is at times a lack of formal code review [18]. Game designers are also expected to have a higher technical mastery, and to know how to integrate different skills, as opposed to the specialization expected of non-game designers. Existing research has not yet documented the inner workings of how game designers use feedback in their work; while game design can be a collaborative process, and designers and developers frequently discuss their work, no formalized understanding exists of the scope of such processes and variations within them.

\section{METHODS}

To better understand the use of peer feedback processes within the game industry we conducted semi-structured interviews with employees at two game development studios in the United States. The first was a mid-sized studio (approximately 50 employees) focused on the mobile market. The second was a smaller studio (approximately 15 employees) primarily focused on contract work for serious game development.

In total we completed 10 interviews from the first studio and 2 from the second. We also recorded video of the workplace and employees' activities at the first. Interviews were an hour long and focused on participants' experiences with peer feedback and playtesting at their company and in their careers in general. The interviews were audio recorded then transcribed. To preserve the anonymity of our participants, we have not identified which participants came from which of the two studios.

Note that, during these interviews, we looked at both peer (between professionals) and user-provided feedback (often in the form of playtests), but the playtest feedback we discuss here comes from internal playtests with participants who are employees of the company. We focus here only on peer feedback, not user feedback.

In order to draw out concrete themes from the data, four research team members each wrote a narrative summary of the interviews they had performed and discussed these within the team. We subsequently parsed the text into a total of 789 chunks, with each describing a single action that participants described doing as part of the development process at their company. A preliminary draft of a process framework emerged through discussion within the team, and this framework was iterated upon an additional two times as data was grouped to fit within steps in the process. As described below, inter-rater reliability tests were subsequently performed with two external coders who were not affiliated with the project. The following section presents general findings from the interviews.

\section{PRELIMINARY FINDINGS}

Across all of the interviews, participants described a number of different forms of feedback in which they engaged. These varied from informal, unscheduled chats to formal meetings involving one or more teams. Participants also discussed different forms of feedback that they received and how helpful each type was in different situations.

\section{Forums for feedback}

In this section we describe the different spaces in which feedback was given or exchanged, whether online or in-person, between individuals or within groups. We also describe particular variants of these types of exchanges that deviate from the standard understanding of the processes.

\section{Informal, drive-by, and virtual feedback}

"I ran into a fellow game designer in the hallway with something I had been thinking about on the project they were working on and we talked in the hallway for 10 minutes about different changes you could make in the game." - P7

The most common form of feedback discussed by participants was in-the moment feedback they solicited from peers. This type of feedback commonly takes the form of casual discussions over chat applications like Slack or Google Hangouts where designers can get quick comments while they work, but broader feedback on ideas or concepts can take place in the halls or even over lunch. These conversations do not always take place between members of the same team; often they are discussions between people in similar roles on different teams.

The slightly more formal variant of this process is when artifacts are uploaded to a shared space, e.g., cloud storage, and peers are asked (or given a "gentle invitation" - P6) to comment when they have time to take a look. This form of feedback is best-suited for situations where small edits or informal ideas can be proposed directly via comment boxes, as opposed to large-scale conceptual feedback that requires a more formal presentation and meeting to discuss. In this case, the creator can asynchronously discuss notes with peers, often by replying to comments. In our data set, this process took place on Quip ${ }^{1}$, a "collaborative productivity software suite" that allows people to create and edit documents and spreadsheets together. This might be used to, e.g., work on wording of scripts or to comment on a design specification document that lays out a plan for future development.

\section{Formal project feedback sessions}

\footnotetext{
${ }^{1}$ https://quip.com/
} 
"If you have a new build that you want to show everybody then that will be in [the regular] Thursday meeting." - P2

These are structured sessions where other members of the studio are invited to a formal meeting to provide targeted feedback on a game, often at weekly or bi-weekly intervals. Attendees are asked to play a build or review a document before arriving and then provide feedback as prompted. During the meeting, one person presents on the project and brings up issues they are having or leads a general feedback session.

The invite-lists of these meetings varied in our data set. In one case, we only heard from interviewees about meetings including designers; other disciplines did not have this type of meeting. In another case, meetings were organized specifically to include non-designer employees with other perspectives, who may see problems in areas where designers have not focused. Throughout our one-on-one interviews these formal meetings were mentioned relatively infrequently.

One particular case of a formal feedback session that deviates from the standard format was a "pitch feedback meeting". This was a new process recently implemented at one of the studios at the time of our visit. Any member of the studio could submit preliminary concepts to an open internal call for ideas. These pitches were then made available for everyone in the studio to read and comment on. The executive team approved a subset of the pitches to move forward, taking these comments into account, and sending additional comments back to the original pitcher to make revisions. The original pitcher might or might not subsequently present a revised version of the pitch in a formal meeting setting.

\section{Team playtests and playtest meetings:}

\section{"We have very explicit processes for internal [playtests] now for the most part ... there are people assigned spe- cific things to play at certain times for tests and they even organized times for people to play sometimes when they don't have time at work." - P3}

These include regular playtesting sessions that are done internally within teams to test out features before release. Members of the team working on the game are expected to devote time to explore new features, often more than once, to help tune them and identify problems. These sessions are often focused on data driven feedback, e.g., regarding completion rates and timing, but qualitative feedback is sometimes collected formally or informally. In our data these were often but not always scheduled at a set time each week so all hands were playtesting simultaneously, which served both to make it a regular occurrence that could be counted on to provide feedback, and to create a sense of cohesion.

An unexpected variant, situated between formal project feedback sessions and team playtests, emerged, which we term "team prototype review meetings". These took place on a team working on an early concept prototype of a game in pre-production. Each member of the team working on the project playtested the game simultaneously in a co-located meeting format, calling out issues as they were encountered. This structure had some interesting benefits as the relevant team member for a given issue was always present to address questions and take note of what would need to be changed.

\section{Types of feedback}

Types of feedback discussed by our participants varied according to what type of forum the feedback was given in and what stage the project was in. These types of feedback are distinct from the three types proposed by Nelson and Schunn described above [19]; all of the types we identify focus on changing the artifact (through the creator) rather than changing the creator in some way.

\section{Conceptual feedback}

Feedback early on in the life of an artifact is primarily conceptual and is frequently given in response to presentations about ideas, as in the case of the "pitch feedback meeting" described above. A concept is presented by its creator, sometimes with slides or as a brief narrative, for the purpose of gaining approval to start work or permission to continue. Comments may be attached to pitches virtually on a shared forum, but when concepts are developed enough to present formally the feedback comes via verbal responses and discussion:

"I think sometimes it's a little slap dash when you have seven people in a room just shouting or ... throwing ideas to the wall and seeing what sticks" - P7

While putting ideas up for open discussion can lead to chaos or wasted time - P1 notes that "Usually we say what we need feedback on, but even then things can get really derailed because people are having ideas" - this back-and-forth is also important because it allows participants to challenge each other and build on each other's ideas in a spontaneous, creative way. The most successful conceptual feedback sessions were those that were managed carefully but not strictly.

Informal feedback on early concepts was rarely mentioned by participants, and was discussed only in the form of a conversation with a respected peer. It is plausible that creators are more personally attached to early concepts because of a slight sense of possessiveness. Therefore, they prefer to have the first round of feedback they receive be from someone they trust to see things at least partially from their perspective:

"There is a product manager I really like to get feedback from because I trust his business sense on one hand, but I also think that he will be direct and won't hold back without being overly critical or cruel about it." - P9

\section{Structural feedback:}

Once a core concept is accepted and more concrete proposals and specifications have been developed, feedback shifts more toward details:

"The first step is the conceptual idea, [but after that] it's writing out the details of how it's gonna work" - P1

During these middle phases of the work, feedback is solicited both through formal meetings and informally through comments on shared materials. It is the job of the presenter in these cases to clearly communicate the characteristics of the 
artifact, whether it is a storyboard, a design specification document, or in another form. It is also their job to communicate what they would like feedback on and what types of feedback are most useful. At this stage, feedback is likely to be on the scale of discussing details of mechanics or game progression, rather than either ideating new general concepts (e.g., critiquing written character dialogue).

As noted above, it is important for feedback in this phase to be both specific and actionable [8, 33], but feedback is also better-received when it is presented in a positive way [20]. To this end, our participants expressed the importance for feedback givers to find a balance between being specific and critical enough to point out areas for improvement while being positive enough to ensure that feedback is well-received:

"I think some people here are probably overly critical sometimes" - P1

"I think sometimes we have a hard time getting that kind of more critical feedback." - P5

Participants in our study often found less-formal feedback via comments or discussion (e.g., on Quip or Slack) preferable to scheduling formal meetings, but it is clear that these two venues serve different purposes. Informal feedback is typically solicited by creators, meaning that creators choose who they want feedback from, which may introduce bias; moreover, participants described informal feedback processes as a way to get feedback on what they were doing, which may lead to a lack of perspective on the bigger picture. Meetings, when constructively run, address both of these issues. Feedback can be exchanged between creators with different backgrounds or specialties or who are working on different aspects of a project. These meetings also facilitate the exchange of integrative feedback, a specific form of structural feedback that connects people working on different pieces of a project and helps improve cohesion between the many parts.

Structural feedback is at the heart of shaping the eventual experience of playing the game. While early conceptual feedback is important in generating novel premises and building a shared vision, structural feedback determines how the game works. In this sense, it helps develop the scaffold upon which features determining user experience will eventually be built and revised. Effective processes for soliciting structural feedback are thus critical for creating a game that is both functional and improve-able in later phases of development.

\section{Play-driven feedback}

"For the playtest data from something that's not released yet, it's very much like how I treat other feedback from [within] the office from peer review... I'll try and see what is the common complaint and then try and not necessarily focus on the specific things they're saying and more [on] why they seem to be saying them." - P3

When an artifact (usually at this stage some piece of a game) is ready for users to engage with it on some level, game designers begin playtesting. This frequently begins with internal playtests within a studio, where members of a team or from various teams will gather to provide feedback on whatever part of a game is being tested. These players' experiences engaging with the game come via "team playtesting" and "playtest meetings", as described above.

Participants in our dataset talked about two different forms of feedback that come from playtests. The first of these is experience-driven feedback on specific features, levels, mechanics, etc. This type of feedback typically includes reactions, opinions, or thoughts building from the player's experiences playing the game, and helps designers consider how they might improve features, choose between alternatives, or scrap particular courses of action. This type of feedback is the most common type of play-driven feedback in early playtests, and remains common until late in the process. This feedback is not always purely qualitative or free-form; playtesters may fill out surveys with numerical scales to rate how much a particular color palette appealed to them or how difficult they found a particular challenge.

"We usually hold a formal playtest session here in the office where we all go sit on the sofas over there. If people have feedback about a particular level or a particular mechanic ... they can give it to us directly and then afterward we can just collate it and make action items and bug reports and things like that." - P7

Note that another form of this type of feedback is identification and reporting of bugs, though these reports are typically less subject to interpretation than comments on experiences.

The second type of feedback participants discussed is quantitative log data automatically gathered during play sessions:

\section{"If we're doing live ops and it's level stuff that I'm look- ing for specifically, I don't even need feedback. I just need plays because we have analytics." - P3}

This data is used to look at things like completion rates, play time, and player choices in order to be able to make decisions about small changes that could be made to the game, even after it has been launched. Participants in our dataset described collecting this type of data primarily from customers, not peers, and as such we suggest that this type of data is not truly a form of peer feedback in its current state.

\section{SIX-STAGE MODEL FOR PEER FEEDBACK IN INDUSTRY GAME DESIGN}

Feedback exchange is not just about the moment of feedback. We present it here as a detailed process, which spans from before the actual feedback exchange and until after the feedback is integrated and used. This process has six stages, with each stage leading directly to the next. Note that the process does not have a concrete beginning or end; it is a cycle, where each time feedback is acted upon (or rejected), the subsequent changes to the artifact will again receive feedback. A designer, developer, artist, or other professional begins with an artifact on which they would like feedback, moves through the process of planning feedback sessions, gathering feedback, reflecting on it, and eventually acting on it, and then returns to the beginning to plan another feedback session. 


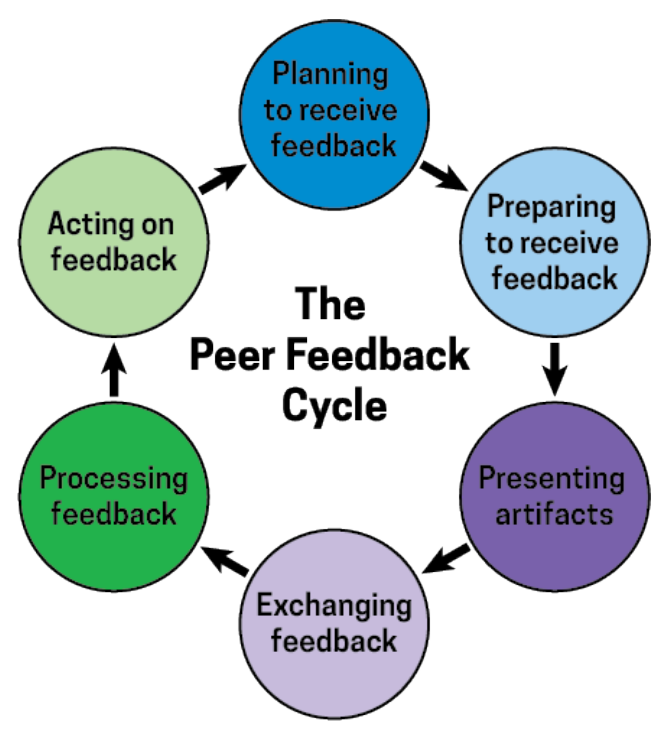

Figure 1. Six-stage model for peer feedback in industry game design

\section{Planning}

Before actual feedback is either given or received, stakeholders plan the feedback process. This includes activities like recognizing that feedback is needed on a particular project; choosing which of the multiple available methods for soliciting feedback should be used; making decisions about who should be involved in giving feedback; and making decisions about the time-line of the feedback exchange.

One challenge of this stage is that the feedback planning process may be initiated by one of several stakeholders. We observed substantial differences based on who initiated the feedback process.

In cases where the feedback receiver initiates the process, they generally have a goal in mind and an artifact on which they want feedback. They see the feedback as a way to help them progress and improve upon their creation, whether because they are blocked or because they want to reflect on their current work. While designers may or may not be able to clearly articulate this goal, the fact that they have initiated feedback planning indicates that they recognize its value.

"Well I try to schedule it so that like people, even if it's usually it's myself and the head of the design research and like the designers of that game specifically will participate" - P2

Feedback planning can also be initiated by feedback providers. When this happens, the feedback provider may not have accurate information about whether the project is ready for feedback; the feedback receiver may or may not agree that feedback is needed; and the feedback receiver may not agree with the process for feedback exchange. However, this type of feedback initiation can be particularly valuable to break creative roadblocks, to avoid perfectionism, or to help designers reflect on their own work. Moreover, as noted above, this type of feedback initiation can lead to integrative feedback; where feedback solicited and received by one individual reacts to their piece of the whole, feedback exchanges initiated by someone with a broader perspective helps keep teams or creators working on different tasks connected and aware of how their pieces fit together.

Finally, third parties (e.g., managers, team leaders) may initiate feedback planning. If the person initiating feedback planning is neither going to receive nor give feedback, it can be experienced as a (perhaps unwanted or inconvenient) obligation for both feedback providers and receivers. However, third parties may have a better sense of the overall needs of the project and the time-line for production, again facilitating opportunities to provide integrative feedback. In our data, third-party initiated feedback exchange sessions were typically regularly scheduled team meetings, though additional feedback sessions were run as necessary when issues arose.

There are several different processes for exchanging feedback, and a core part of feedback planning is choosing which of these is best for the circumstances. Different feedback processes have different strengths and weaknesses, both in terms of feedback quality and in terms of how difficult the process is to initiate and execute. Across our data set, we observed that designers are highly sensitive to the burden of initiating and planning a feedback process, particularly including the social costs; while all designers are aware of the time costs of soliciting feedback, some also perceived initiating a feedback exchange, particularly informally, to be akin to asking for a favor. Additionally, designers do not always consider all the feedback options available to them when initiating feedback planning - a classic choice overload problem. Designers have broad categories for differentiating the type of feedback they receive (e.g. conceptual versus play-driven) and for identifying the purpose of the feedback (e.g. reactions to pitches versus iterating on features), but they seem less able to articulate other relevant factors such what form the feedback needs to be in order to be most helpful (i.e., level of depth, specificity, actionability $[8,26,33])$. Providing designers with process selection may therefore be a useful investment of effort.

A final challenge we observed is social obligation. Part of the feedback process involves inviting others to participate, either as feedback receivers or feedback providers. These invitations can create a sense of obligation in one of two ways. First, there can be overt power at play. For example, being asked to participate in a feedback process by a direct superior does not always offer a way out. Second, people may feel a sense of personal obligation, particularly if they are being asked by someone with whom they have a positive prior relationship. Social obligation can impact feedback both positively and negatively. It can encourage people to produce higherquality feedback via peer pressure or social facilitation effects [37], but when power dynamics or personal relationships are at stake, feedback providers may feel pressure to give more positive feedback than they feel is honestly deserved. Providing feedback that is critical, even if it is both warranted and welcome, can feel risky to participants. Though anonymity mitigates some of this sense of risk, it also can lead to decreased quality of feedback, particularly over time [15]. 
While there are ways to deal with this issue at other times in the feedback process (see below), there are several ways to manage it during the planning process. For example, expectations for when and how it is appropriate to ask for informal types of feedback can help mitigate social pressure. Encouraging mutual rather than one-way feedback sessions, when appropriate, can also help, as long as it is clear that all participants are expected to give feedback according to the recipient's needs.

\section{Preparing}

After making plans to receive or exchange feedback, receivers start to make necessary preparations. The main decisions that need to be made in this stage include selecting which materials need to be tested and/or reviewed, what method should be used to collect feedback, and how to share or present the materials. Once the feedback-receiver has decided what they need feedback on, they may be responsible for logistics such as planning meetings; this may require working with peers to find a space in busy schedules that allows for adequate review of materials in advance. The receiver may send feedback providers the materials ahead of time or grant them access. For instance, receivers may ask providers to review the build that they are trying to test. In tandem with sharing materials, the receiver must also convey their expectation of what the providers should prepare before the session, e.g., in the case of testing a build, whether the providers need to play it before the meeting.

"I send them a build via a link... I post that in slack, send out an email, announce it at the company meeting and then create a document that anyone could have access to that they could write their feedback on." - P9

The feedback providers' most important responsibility is to understand and keep to the set time-line, reviewing materials ahead of time as needed and allowing time to reflect on their experiences before giving feedback. This may include writing preliminary feedback in a document ahead of a meeting, as in P9's quote above, or jotting down notes for what they plan to discuss in the meeting.

These preparations vary significantly according to which forum for feedback is used. In the case of informal feedback during a conversation in the hallway, little to no preparation may be required from either party. If feedback is to be provided via formal project feedback sessions, expectations may be the same for every regularly scheduled meeting with interim deadlines for feedback-receivers to submit prepared materials and for providers to review the materials. In the case of team playtests, it may be better for feedback providers not to review materials ahead of time in order to provide more natural, in-the-moment reactions.

Primary challenges in this stage are logistical and scheduling difficulties as well as the possibility of inadequate or rushed preparation from either side. The artifact (e.g., a playable build) provided by the receiver may not be completely ready when needed due to rushed processes or tight deadlines. Moreover, feedback receivers may not have taken adequate time to consider what types of feedback would be most useful for them:

\section{"The biggest problem is probably when we aren't really sure exactly what we're looking for in a playtest" - P3}

Providers may also not have time to review the artifact in depth before a meeting, leading them to provide shallow feedback or feedback based on an incorrect understanding of the artifact. As above, effective scheduling processes help mitigate the impact of these issues, as does prompt and honest communication about when one has not had time to complete expected preparations. Flexibility of the format or forum for feedback exchange may also address some of these challenges. If an artifact is unlikely to be ready for feedback at a scheduled meeting, the ability to switch to informal, asynchronous, comment-based feedback can help avoid wasted time.

\section{Presenting}

Presenting is characterized by sharing of material on which feedback is wanted, or viewing of material on which feedback is requested.

Once a feedback recipient has established the format and forum for feedback and adequately prepared the artifact, they then present the artifact to feedback providers in some form. Details set in the two preceding stages come to life here. If feedback is solicited informally, this presentation may take the form of a quick discussion or a few messages back and forth on Slack; if a formal project feedback session has been chosen as the appropriate forum, presenters may speak for a set period of time in front of a set of slides or show the artifact; if feedback comes via team playtesting, the presentation takes the form of testers playing the game, sometimes with guidance from the "presenters". The flow of the presentation (or lack thereof) is dictated by planning done before this stage.

\section{" We're very visual here and it seems like if you have a visual it's very impressive and ... it's the same thing with our pitch process. It's like you need a lot of ... you almost need slides and stuff because if you just look right at dry document, people might not even [engage]." - P6}

In this stage, the primary challenges are again related to communication. The quality of feedback received depends directly on how well the presenter communicates both what they have done and what type of feedback they would find most useful. Though developing a detailed and accessible presentation may seem to be a waste of time when this time could be spent actually improving the artifact, presenters should note that giving a poor presentation leads to a waste of the time of the feedback providers who lose the ability to contribute usefully.

\section{Exchanging}

In the Exchanging stage, feedback goes from providers to receivers. Feedback providers and receivers may shift roles during a meeting, as in the case where multiple people present over the course of a meeting and each gives feedback on the others' work. In this stage, presenters take notes on the feedback given and respond to feedback with follow-up questions as appropriate. They may also distribute additional materials for evaluation, such as surveys, or may pose specific questions that they want feedback on. 
"We'd all play it in a space together and then just [give] free form [feedback], just anything that's coming up. If we were looking for a specific stuff we'd highlight it ... As somebody that was maybe running one of those, I'd just have a notebook with me and just be writing down notes like crazy. - P5

There are various decisions that needed to be made for this stage to happen: where the exchange happens; whether the feedback should be anonymized or the location made more private; whether feedback should be recorded, and if so, through what medium; and whether the presentation and feedback exchange will occur in real time or asynchronously.

Social dynamics factor significantly into the success of this phase. Beyond getting the right people in the room (or on the virtual comment thread), which can in itself be a significant challenge, the relationships between presenter(s) and feedback providers will shape the outcomes of the feedback session. If the presentation is given in a co-located space, conversational styles may clash if not carefully managed; certain feedback providers may tend to dominate conversations or may be rigid in their biases about particular approaches or concepts.

"It can be trickier because some people will either start going along with what other people are saying even if it's not what they're thinking because of social pressures or there are certain people that don't like talking in front of groups and so they may not say things they're thinking even though they have opinions." - P3

Similarly, presenters may be variably resistant to feedback; some presenters who are deeply invested in what they have created or who take feedback personally may be more resistant to given feedback, but others with less self-confidence may feel obligated to accept all feedback uncritically.

"I've seen a lot of people who are junior often have problems with either ignoring all of it or listening to all of it too much" - P3

Effective managers may be able to mitigate these issues, but it is also the responsibility of the presenter to shape the feedback session to be most helpful to them.

\section{Processing}

"The [next] thing I was doing was processing all that information and then coalescing it all into good action items and takeaways of ways that we can improve the game that we're working on." - P5

During this stage, the receivers process and understand the information that they received from the exchange stage. The feedback received from previous stage will be collected and organized. The receivers at this time will often decide to process the information individually or discuss and understand with team members. While going through the feedback and trying to make sense of it, the receiver will decide what information is important and should be adopted to improve the current product. This stage mainly involves the receivers; providers' responsibilities mainly include availability for follow-ups when necessary.
Decisions that get made during this stage primarily focus on how to understand and make use of the feedback. These decisions include whether to process the feedback individually or with team members; what feedback to consider and what to disregard; whether the feedback requires a follow-up; whether the feedback is immediately actionable; and whether the feedback provider has adequate expertise. Summation and interpretation of feedback is done primarily through individual reflection or group discussion. No formal processes beyond these were shared by our participants.

Though no explicit problems during this stage were reported by participants, the current lack of formal approaches to reflection presents options for exploring the use of exercises or practices to mitigate some of the biases that may exist. Interpretation of feedback is flexible, and creators who try to look at the "spirit" behind feedback may see what they want to see rather than what has been said.

\section{Acting}

Acting is characterized by executing on decisions made in the processing stage. Changes may be made on parts, the whole, or none at all. The game may continue to exist in its current form or slightly modified:

"So basically I ended up redesigning the app icon to match more with the visual style but still have some of the heart that the concept that I came up with beforehand [had]." - P4

Or in some cases it may be scrapped completely:

"Occasionally we throw it all out and just completely change everything based on feedback, and that is hard when that happens, but it does happen." - P4

This stage contains the processes of development that occur between reflecting on feedback and planning to receive feedback on a more-developed artifact. Based on what they take from the feedback, designers revisit what they had previously created and modify or continue to improve it in relevant ways. Many of designers' day-to-day tasks will fall within this stage.

Though we emphasize that the process we have described is a cycle, we begin our description with feedback planning and end with development in order to emphasize the core role of seeking and exchanging feedback in the design process. Design is not a single arc of development, feedback, and revision; feedback on a design drives revision, which drives the next set of feedback in return. Except for in the rare case where a single person designs and develops a game completely alone without feedback from anyone, peer feedback is integrated into design from the very beginning. Even the initial pitches for new games or concepts are driven on some level by feedback on prior ideas in related contexts. Games exist within this cycle from the initial ideation phases up until all iteration is complete, often well after they have been put into production.

\section{MODEL VALIDATION AND LIMITATIONS}

In order to validate the model we present above, we performed inter-rater reliability testing. Two independent qualitative coders were given the codebook shown in Table 1 listing the 


\begin{tabular}{l|l}
\hline Name of Stage & Stage Descriptions \\
\hline Planning to receive feedback & $\begin{array}{l}\text { Decisions are made, including identifying that feedback is needed, when } \\
\text { feedback is to occur, who is invited to participate. }\end{array}$ \\
\hline Preparing to receive feedback & $\begin{array}{l}\text { Executing concrete actions to prepare for feedback. This includes creation and } \\
\text { sending off of surveys or non-disclosure agreements, preparing slides for a } \\
\text { presentation, uploading or sharing files. }\end{array}$ \\
\hline Presenting artifact & $\begin{array}{l}\text { Sharing of material on which feedback is wanted, or viewing of material on } \\
\text { which feedback is requested. }\end{array}$ \\
\hline Processing feedback & $\begin{array}{l}\text { Feedback goes from givers to receivers. Givers and receivers may or may not } \\
\text { shift roles in a same meeting. }\end{array}$ \\
\hline Acting on feedback & $\begin{array}{l}\text { Feedback received is digested by the receivers, and decisions are made. In- } \\
\text { cludes collating, revising, value judgement. }\end{array}$ \\
\hline & $\begin{array}{l}\text { Executing on decisions made. Changes may be made on parts, the whole, or } \\
\text { none at all. The game may continue to exist or be scrapped completely. }\end{array}$ \\
\hline
\end{tabular}

Table 1. Six-stages of peer feedback model and descriptions

six stages and brief descriptions. We chose coders who are not involved in this project and who were not previously aware of it in order to mitigate our own biases and subconscious mutual understandings of the meaning behind the codes. This framework is intended to be suitable for application by anyone with knowledge of game design or peer feedback, and as such we performed this testing to show that it could be used by others outside the research team.

Both coders were assigned the same $10 \%$ of the overall dataset, and each coder independently assigned one of the six phases to each chunk of text drawn from the interview transcriptions. We then compared the codes each rater had assigned to each chunk with each other through calculation of a Cohen's $\kappa$ coefficient, a commonly-used inter-rater reliability statistic. We found $\kappa$ $=0.53$, or moderate agreement between raters. However, Cohen's $\kappa$ is traditionally used when raters are grouping discrete, nominal categories; in this case, phases are ordinal, meaning that there is a sequence. If one rater labels a stage "Processing feedback" and the other labels it "Acting on feedback", this is closer to agreement than if the second had instead labeled it "Presenting artifact". Association between two raters who are using ordinal scales is traditionally calculated with Spearman's $\rho$ correlation. When we calculated this correlation with our dataset we found a $\rho=0.72$, indicating moderate to strong agreement.

Note that even Spearman's $\rho$ does not account for cyclical data. In our sample this meant that if one coder labeled a chunk "Acting on feedback" and the other labeled it "Planning to receive feedback", this was treated as significant disagreement Spearman's $\rho$ calculation, as the steps are adjacent in the cycle but appear far apart if the cycle is represented as a sequence with a beginning and an end. Though there are not widely-used inter-rater reliability statistics that take into account cyclical ordinal data, we suggest that our statistics would be even stronger if we were able to include this aspect of the model in calculations.

The primary limitation of this work is that it is based on data collected from two studios, which cannot represent the entire space of game development practices. Our sample did not contain perspectives from very small indie studios, where the notion of a peer is likely very different, or very large first-party developers, where team sizes are much larger. Further validation of this model based on surveys of game designers and interviews with a wider population is necessary to understand how the steps described here vary across different types of studios. Future work could improve on the model by clearly establishing how size of studio impacts each of these phases.

\section{DISCUSSION AND IMPLICATIONS}

This framework is intended not only as a synthesis of what we observed but also as a starting point to drive interventions, moving beyond how the process of peer feedback works toward whether and how it can altered. Interventions could be targeted toward specific phases within this process or could aim to shift the process in its entirety. Similarly-structured frameworks have already been used to drive interventions within game design processes, as in the case of playtest feedback in game design classes [13].

Interventions could vary across a number of dimensions. We pick three dimensions here as examples to provoke discussion: formal vs informal, digital vs analog, and individual vs group. These interventions could be designed as technologies, social interventions, or as part of a broader socio-technical system.

In order to demonstrate the potential applications for the peer feedback cycle we present, we consider as an example the commonly-known challenge of being "helpfully critical" in feedback-giving. As noted above, we observed problems in our dataset with feedback providers both being unwilling to be critical and also being critical in unhelpful ways, e.g., being critical of the person rather than the product. Similarly, some designers are less willing to listen to critical feedback. The following are six potential interventions to address this challenge, with one corresponding to each stage in the cycle:

During the planning stage, when feedback receivers are deciding who to include in a potential feedback exchange, they could consult digital feedback portfolios that track the feedback others have given in the past. Such portfolios could also serve as a record of professional contributions - being able to point to concrete pieces of feedback that they had given that led to improvements in a peer's work would help professionals demonstrate their value beyond what they create themselves. 
An individual-focused intervention in the preparation stage could be development of training sessions to help feedback providers learn how to give more helpful feedback, as tailored to the needs of the studio's projects. These could draw on some of the lessons from research into effective feedbackgiving practices outlined above.

To handle the presentation stage, physical artifacts could be developed to indicate when certain types of interruptions were permissible during presentations. These could help avoid questions that derail the presentation while ensuring that clarifications are made when necessary.

In the exchanging stage, a group-focused set of guiding questions could be given to help direct people to give better, more effectively-critical feedback, similar to the process used in the PeerPresents system [27].

In the processing stage, in order to help feedback receivers screen feedback, a formalized process could be developed to identify characteristics of important pieces of feedback in order to ensure that they are not missed or resisted due to the form of critique. Ambrosino et al. [1] present a conceptuallysimilar process for screening ideas during a design process, which could be adapted to screen feedback.

One of the challenges of the acting stage is knowing when enough revision has been completed to make it useful to proceed again to planning. An informal set of guidelines or selfcheck-ins could be established to prompt creators to consider at regular intervals whether feedback could be useful.

\section{Assessing outcomes}

Providing support for different stages of the peer feedback process could positively impact designers' work in multiple ways. Perhaps most obviously, improving feedback processes could lead to creating better games, potentially in less time, realizing tangible efficiency gains in development. Alternatively, improving how design teams support each other through feedback could have implications on designers' job satisfaction or happiness and team morale more broadly. A culture of creators who are comfortable with giving each other regular feedback and framing it to be critical, specific, actionable, and considerate would be a strong asset for any studio.

Though this peer feedback model could generalize to apply in other contexts, we believe that it is particularly relevant to the game industry for several reasons. First, game design is situated in the overlap between art and engineering, with creativity being of paramount importance. The research on creativity shows that while individuals may come up with more diverse ideas when working alone, the highest quality ideas come from interactions rather than individuals [25]. Second, game design is a notably interdisciplinary process. Designers often have to assume multiple roles that require different competencies; similarly, people who are not expert in a particular domain (e.g. art) must engage with that domain to provide feedback in areas where they are expert. In cases like these, studios benefit when expertise can be shared rapidly and effectively. Finally, the constant cycles of revision within game design, often extending well after games have been published, make it an extremely iterative process, and iteration builds both from individual reflection and from feedback.

If feedback processes are to be improved upon, we must also consider what to assess to determine whether and how a process improves when an intervention is made. A number of dimensions could be measured. Most obvious among these is product quality - though each studio likely has different strategies in measuring how good the games they develop are, effective peer feedback processes should lead to better games. Second, designers and developers should demonstrably improve their understanding of each other's disciplines and the work happening in other parts of the development process. This improved understanding will also contribute to individuals' professional development. Third, making regular exchange of constructive peer feedback the norm contributes to a more positive workplace culture overall, leading to increased satisfaction and stronger identification with teams and the studio as a whole.

\section{CONCLUSION}

Though the model we present here represents the full peer feedback process that we observed, it is intended as a starting point for further work and development in this space. As these findings are based on findings from small and mid-sized studios, one of the first steps in further validating this framework is to engage with other sizes and types of studios and designers, whether through further interviews or a broader survey of professionals in the game industry. Possible questions to explore within this work include how much time designers spend on each of these stages, how this varies across environments, and whether and how technical, social, and administrative support during each phase impacts success. Improving our understanding of the peer feedback process in professional settings also has pedagogical implications, as we can compare differences between how this process is taught and how it is actually performed.

Future work could also explore the effectiveness of interventions, perhaps building from the examples described above. Given that peer feedback is by definition socially situated, the differential impacts of these kinds of interventions across different sizes and types of studios would be important to study.

Broadly, with this work we hope to drive further research into peer feedback in game design, as noted above, but also to help designers examine their own processes for getting peer feedback. The model we propose here serves both as a summary of findings from the interviews we performed and a starting point for future exploration.

\section{ACKNOWLEDGMENTS}

We would like to thank Amazon for funding that supported this project. We would also like to thank the NSF for funding this work under NSF Grant \#1821590. Finally, we would like to thank our participants for contributing their time. 


\section{REFERENCES}

[1] Julien Ambrosino, Dimitri Masson, Audrey Abi Akle, and Jérémy Legardeur. 2017. Fostering collaborative project emergence through divergence of opinion. In ICED '17, Vol. 8. The Design Society, 489-498.

[2] Niccolo' Becattini, Cedric Masclet, Fatma Ben Guefrache, Guy Prudhomme, Gaetano Cascini, Elies Dekoninck, and others. 2017. Characterisation of a co-creative design session through the analysis of multi-modal interactions. In ICED '17. The Design Society, 479-488.

[3] Jessica L Bienstock, Nadine T Katz, Susan M Cox, Nancy Hueppchen, Sonya Erickson, Elizabeth E Puscheck, and others. 2007. To the point: medical education reviews - providing feedback. American journal of obstetrics and gynecology 196, 6 (2007), 508-513.

[4] Kun Hung Cheng, Jyh Chong Liang, and Chin Chung Tsai. 2015. Examining the role of feedback messages in undergraduate students' writing performance during an online peer assessment activity. Internet and Higher Education 25 (2015), 78-84. DOI :

http://dx.doi.org/10.1016/j.iheduc. 2015.02.001

[5] Judeth Oden Choi, Jodi Forlizzi, Michael Christel, Rachel Moeller, MacKenzie Bates, and Jessica Hammer. 2016. Playtesting with a Purpose. In Proceedings of the 2016 Annual Symposium on Computer-Human Interaction in Play - CHI PLAY'16. ACM, 254-265. DOI : http://dx.doi.org/10.1145/2967934.2968103

[6] Nigel Cross. 2011. Design thinking: Understanding how designers think and work. Berg.

[7] Sabrina Culyba. 2018. The Transformational Framework: A Process Tool for the Development of Transformational Games. ETC Press.

[8] Anne Dohrenwend. 2002. Serving up the feedback sandwich. Family practice management 9, 10 (2002), 43.

[9] Tracy Fullerton, Chris Swain, and Steven Hoffman. 2004. Game design workshop: Designing, prototyping, \& playtesting games. CRC Press.

[10] AndrÃl' Godoy and Ellen F Barbosa. 2010. Game-Scrum: An Approach to Agile Game Development. In Proceedings of SBGames 2010. SBGames.

[11] Ulf Hagen. 2010. Designing for player experience. In Nordic DiGRA. Citeseer.

[12] Ulf Hagen. 2011. Designing for player experience: How professional game developers communicate design visions. Journal of Gaming \& Virtual Worlds 3, 3 (2011), 259-275.

[13] Jessica Hammer and Amy Cook. 2018. EOTA: A Method for Improving Peer Feedback in the Game Design Classroom. In International Academic Conference on Meaningful Play.
[14] Bruce Hanington and Bella Martin. 2012. Universal methods of design: 100 ways to research complex problems, develop innovative ideas, and design effective solutions. Rockport Publishers.

[15] Yasmine Kotturi, Andrew Du, Scott Klemmer, and Chinmay Kulkarni. 2017. Long-Term peer reviewing effort is anti-reciprocal. In Proceedings of the Fourth (2017) ACM Conference on Learning@ Scale. ACM, 279-282.

[16] Jill Larkin, John McDermott, Dorothea P Simon, and Herbert A Simon. 1980. Expert and novice performance in solving physics problems. Science 208, 4450 (1980), $1335-1342$.

[17] Bryan Lawson. 2012. What designers know. Routledge.

[18] Emerson Murphy-Hill, Thomas Zimmermann, and Nachiappan Nagappan. 2014. Cowboys, ankle sprains, and keepers of quality: How is video game development different from software development?. In Proceedings of the 36th International Conference on Software Engineering. ACM, 1-11.

[19] Melissa M Nelson and Christian D Schunn. 2009. The nature of feedback: How different types of peer feedback affect writing performance. Instructional Science 37, 4 (2009), 375-401.

[20] Thi Thao Duyen T. Nguyen, Thomas Garncarz, Felicia Ng, Laura A. Dabbish, and Steven P. Dow. 2017. Fruitful Feedback. In Proceedings of the 2017 ACM Conference on Computer Supported Cooperative Work and Social Computing - CSCW'17. ACM, 1024-1034. DOI : http://dx.doi .org/10.1145/2998181.2998319

[21] Melissa M. Patchan, Christian D. Schunn, and Russell J. Clark. 2017. Accountability in peer assessment: examining the effects of reviewing grades on peer ratings and peer feedback. Studies in Higher Education 5079, May 2017 (2017), 1-16. DOI :

http://dx.doi .org/10.1080/03075079.2017.1320374

[22] Cristiano Politowski, Lisandra Fontoura, Fabio Petrillo, and Yann-GaÃńl Guéhéneuc. 2016. Are the old days gone? A Survey on Actual Software Engineering Processes in Video Game Industry. In GAS '16. ACM. DOI : http://dx.doi .org/10.1145/2896958.2896960

[23] Rido Ramadan and Yani Widyani. 2013. Game Development Life Cycle Guidelines. In 2013 International Conference on Advanced Computer Science and Information Systems (ICACSIS) : Sanur Paradise Plaza Hotel, Bali, September 28th-29th, 2013. IEEE.

[24] Katie Salen, Katie Salen Tekinbaş, and Eric Zimmerman. 2004. Rules of play: Game design fundamentals. MIT press.

[25] Keith Sawyer. 2017. Group genius: The creative power of collaboration. Basic books. 
[26] Amy Shannon, Jessica Hammer, Hassler Thurston, Natalie Diehl, and Steven Dow. 2016a. PeerPresents: A Web-Based System for In-Class Peer Feedback during Student Presentations. Proceedings of the 2016 ACM Conference on Designing Interactive Systems - DIS '16 (2016), 447-458. DOI :

http://dx.doi.org/10.1145/2901790.2901816

[27] Amy Shannon, Jessica Hammer, Hassler Thurston, Natalie Diehl, and Steven Dow. 2016b. PeerPresents: A web-based system for in-class peer feedback during student presentations. In Proceedings of the 2016 ACM Conference on Designing Interactive Systems. ACM, 447-458.

[28] Amy Shannon, Alex Sciuto, Danielle Hu, Steven P. Dow, and Jessica Hammer. 2017. Better Organization or a Source of Distraction?. In Proceedings of the 2017 CHI Conference on Human Factors in Computing Systems CHI'17. ACM, 5545-5555. DOI : http://dx.doi.org/10.1145/3025453.3025564

[29] Liridona Sopjani, Mia Hesselgren, Sofia Ritzén, Jenny Janhager Stier, and others. 2017. Co-creation with diverse actors for sustainability innovation. In ICED ' 17 , Vol. 8. The Design Society, 459-468.

[30] Kurt Squire, Mike Barnett, Jamillah M Grant, and Thomas Higginbotham. 2004. Electromagnetism supercharged!: learning physics with digital simulation games. In Proceedings of the 6th international conference on Learning sciences. International Society of the Learning Sciences, 513-520.

[31] Thomas Staubitz, Dominic Petrick, Matthias Bauer, Jan Renz, and Christoph Meinel. 2016. Improving the Peer Assessment Experience on MOOC Platforms. In Proceedings of the Third (2016) ACM Conference on Learning@Scale-L@S'16. ACM, 389-398. D0I: http://dx.doi.org/10.1145/2876034.2876043
[32] Yassine Talas, Lilia Gzara, Marie-Anne Le Dain, Valéry Merminod, and Alejandro Germán Frank. 2017. Which are the limitations of ICT tools for collaborative design with suppliers?. In ICED '17. The Design Society.

[33] Jane DeLima Thomas and Robert M Arnold. 2011. Giving feedback. Journal of palliative medicine 14, 2 (2011), 233-239.

[34] F. Ted Tschang. 2007. Balancing the Tensions Between Rationalization and Creativity in the Video Games Industry. Organization Science 18, 6 (2007), 989-1005. DOI : http://dx.doi.org/10.1287/orsc. 1070.0299

[35] Pamela V Ulrich, Lenda Jo Anderson-Connell, and Weifang Wu. 2003. Consumer co-design of apparel for mass customization. Journal of Fashion Marketing and Management: An International Journal 7, 4 (2003), 398-412.

[36] Mark E. Whiting, Freddie Vargus, Tejas Seshadri Sarma, Varshine Chandrakanthan, Teogenes Moura, Mohamed Hashim Salih, Gabriel Bayomi Tinoco Kalejaiye, Adam Ginzberg, Catherine A. Mullings, Yoni Dayan, Kristy Milland, Dilrukshi Gamage, Henrique Orefice, Jeff Regino, Sayna Parsi, Kunz Mainali, Vibhor Sehgal, Sekandar Matin, Akshansh Sinha, Rajan Vaish, Michael S. Bernstein, Snehalkumar (Neil) S. Gaikwad, Aaron Gilbee, Shirish Goyal, Alipta Ballav, Dinesh Majeti, Nalin Chhibber, and Angela Richmond-Fuller. 2017. Crowd Guilds: Worker-led Reputation and Feedback on Crowdsourcing Platforms. In Proceedings of the 2017 ACM Conference on Computer Supported Cooperative Work and Social Computing - CSCW'17. ACM, 1902-1913. DOI: http://dx.doi .org/10.1145/2998181.2998234

[37] Robert B Zajonc. 1965. Social facilitation. Science 149, 3681 (1965), 269-274. 